\title{
Covid-19 e coordenação federativa no Brasil: consequências da dissonância federal para a resposta à pandemia
}

\author{
Covid-19 and federative coordination in Brazil: consequences of \\ federal dissonance for the pandemic response
}

Fabiola Sulpino Vieira', Luciana Mendes Santos Servo ${ }^{\mathbf{1}}$

DOI: 10.1590/0103-11042020E406

RESUMO Este ensaio teve por objetivo discutir a trajetória da coordenação federativa no Sistema Único de Saúde (SUS) e a coordenação dos esforços de resposta à pandemia de Covid-19. Para dar suporte à discussão, aborda referencial teórico sobre a relação entre o federalismo e a implementação de políticas públicas, e sobre o desenvolvimento da coordenação federativa no SUS. Também discute decisões recentes do Ministério da Saúde que resultaram na fragilização dos instrumentos de coordenação do sistema. Pondera que a coordenação federal deficiente na resposta à pandemia não se deve à ausência de mecanismos de coordenação federativa do SUS ou ao constrangimento desses mecanismos impostos pelos demais entes da federação. O que se observa é a deliberada fragilização desses instrumentos pelo governo federal. Conclui que a crise sanitária acelerou o processo de distanciamento do Ministério da Saúde do seu papel de dirigente nacional do SUS e explicitou a decisão do governo federal pela inação, relegando ao órgão papel secundário nos esforços de enfrentamento da pandemia, com graves consequências para o acesso da população aos cuidados de saúde, especialmente os intensivos, e para a efetividade das ações no campo da vigilância em saúde.

PALAVRAS-CHAVE Federalismo. Acesso aos serviços de saúde. Vigilância em saúde pública. Pandemias. Coronavírus.

\begin{abstract}
This essay aimed to discuss the trajectory of federative coordination in the Unified Health System (SUS) and the coordination of response efforts to the Covid-19 pandemic. To support the discussion, it addresses a theoretical framework on the relationship between federalism and the implementation of public policies, and on the development of federative coordination in the SUS. It also discusses recent decisions by the Ministry of Health that resulted in the weakening of the system's coordination instruments. It considers that the deficient federal coordination in the response to the pandemic is not due to the absence of federative coordination mechanisms of SUS or to the constraint of these mechanisms imposed by the other entities of the federation. What is observed is the deliberate weakening of these instruments by the federal government. It concludes that the health crisis accelerated the process of distancing the Ministry of Health from its role as national director of SUS and made explicit the decision of the federal government for inaction, relegating to the Ministry a secondary role in efforts against the pandemic, with serious consequences for access population to health care, especially intensive care, and for the effectiveness of actions in the field of health surveillance.
\end{abstract}

KEYWORDS Federalism. Health services accessibility. Public health surveillance. Pandemics. Coronavirus. 


\section{Introdução}

A implementação de medidas de enfrentamento da pandemia de Covid-19 desafiou os maiores e mais bem preparados sistemas de saúde do mundo. Se por um lado eles já possuíam grande experiência em lidar com emergências de saúde pública, por outro, o desconhecimento da história natural da doença, a falta de tratamento específico e as particularidades da infecção pelo novo coronavírus (SARS-CoV-2) impuseram desafios à resposta dos países à pandemia. A velocidade de transmissão, o número de reprodução (número de infecções secundárias geradas por um indivíduo infectado) e a gravidade da Covid-19 são maiores do que os observados até então para as gripes causadas pelos diferentes subtipos do vírus Influenza ${ }^{1}$.

No Brasil, houve um aumento expressivo de hospitalizações por Síndromes Respiratórias Agudas (SARG) em 2020, comparado com anos anteriores ${ }^{2}$, sendo isso indicativo de subnotificação da Covid-19. Paralelamente, os óbitos por pneumonia e insuficiência respiratória apresentaram aumento em São Paulo, Rio de Janeiro e Manaus, quando essas capitais registravam o maior número de casos da doença ${ }^{3}$.

Somaram-se às dificuldades mencionadas, a crise política que se prolonga há alguns anos e que se agravou nos últimos meses; as fragilidades do Sistema Único de Saúde (SUS), decorrentes de anos de baixa prioridade política e de subfinanciamento crônico ${ }^{4,5}$; e a atuação dissonante do governo federal em relação ao trabalho de enfrentamento da pandemia realizado pelos demais entes da federação ${ }^{6}$, na contramão das boas práticas informadas por evidências científicas.

A respeito dessa última questão, foi explícita a posição contrária do presidente da República às medidas de distanciamento social horizontal $^{6}$, as quais foram reconhecidas como as mais efetivas para a contenção da transmissão do coronavírus ${ }^{7}$. Também foram irrestritas as suas críticas aos governadores e prefeitos que adotaram tais medidas, atribuindo a eles a responsabilidade pela recessão econômica que se seguirá à crise sanitária ${ }^{8}$, em uma contraposição entre economia e saúde. Ademais, sua decisão para que o Ministério da Saúde (MS) recomendasse o uso amplo de cloroquina e hidroxicloroquina no tratamento da Covid19 resultou na queda de dois ministros ${ }^{9}$ e na publicação de uma 'orientação' sobre o uso precoce desses medicamentos no tratamento da doença ${ }^{10}$, a despeito de as evidências científicas não embasarem a sua indicação nesse caso até o momento"1.

Os embates do presidente da República com governadores e prefeitos levaram à judicialização do tema, com decisão do Supremo Tribunal Federal (STF) de que a competência da União, dos estados, do Distrito Federal e dos municípios é concorrente em matéria de saúde e que, portanto, todos os entes da federação têm autonomia para tomar providências normativas e administrativas relacionadas à Covid-1912. Entretanto, essa decisão não pôs fim às divergências ou gerou espaço para uma ampliação da coordenação federativa no enfretamento da pandemia.

Segundo Abrucio ${ }^{\text {13(41), }}$, a coordenação federativa ou intergovernamental diz respeito às "formas de integração, compartilhamento e decisão conjunta presentes nas federações". Trata-se de aspecto essencial que visa garantir o equilíbrio entre a autonomia dos entes e a interdependência entre eles ${ }^{\mathbf{1 4}}$, o qual é determinante para o enfrentamento da presente crise sanitária. Na resposta à Covid-19 pelos países, as seguintes atividades são apontadas como essenciais: i) coordenação e consistência nas ordens para ficar em casa em todas as jurisdições; ii) rápida testagem para identificação do novo coronavírus; e iii) melhoria da capacidade de resposta do sistema de saúde ${ }^{15}$.

Considerando a atual crise sanitária e a necessária articulação entre o MS e as secretarias de saúde estaduais e municipais, o objetivo deste ensaio é discutir a trajetória da coordenação federativa no SUS e a coordenação dos esforços de resposta à pandemia de Covid-19, com foco especial na atuação do 
governo federal e nas consequências dessa atuação para o funcionamento do sistema, o atendimento das necessidades de saúde da população brasileira e a efetividade das ações de enfrentamento da doença no País.

A discussão é fundamentada por achados da literatura científica e por informações constantes de documentos legais. Dado que muitos acontecimentos são extremamente recentes e que não foram ainda reportados em documentos técnico-científicos, utilizaram-se matérias jornalísticas como fonte de informação, uma vez que nelas se encontram o registro de fatos relacionados com o tema ora tratado.

O ensaio é constituído de outras cinco seções além desta introdução, nas quais se busca tratar da relação entre o federalismo e a implementação de políticas públicas; abordar a coordenação federativa no SUS em uma perspectiva histórica; discutir as mudanças recentes que afetaram instrumentos de coordenação do SUS em nível federal; refletir sobre as consequências dessas alterações e da conjuntura política atual para a resposta brasileira à pandemia; e apresentar, em síntese, nas considerações finais, as questões-chave discutidas neste ensaio.

\section{Federalismo e políticas públicas}

$\mathrm{O}$ federalismo pode ser entendido como uma forma de organização do poder do Estado com um nível de governo central, de caráter nacional, e outro descentralizado, constituído pelos governos subnacionais, em que ambos os níveis têm autonomia territorial, e os representantes de cada governo têm poderes exclusivos e concorrentes sobre o mesmo território e população ${ }^{16}$; e as políticas públicas são programas e ações governamentais com impactos sobre a população, que traduzem os propósitos dos governos ${ }^{17}$.

O tema 'federalismo e políticas públicas' tem sido tratado em vasta literatura dos campos da economia, da ciência política e da saúde ${ }^{16}$. $\mathrm{O}$ interesse de distintos campos do conhecimento nesse assunto pode ser explicado, em parte, pela necessidade de compreender os efeitos das relações entre os entes federados sobre o processo de implementação de políticas públicas, especialmente as de abrangência nacional, em situação de descentralização da competência normativa e administrativa em matérias específicas, como saúde e educação. Há muitas definições para o termo descentralização, mas uma, bastante objetiva, é a de que se trata de uma mudança na alocação da autoridade, de forma que o poder é deslocado para unidades territoriais menores de governo ${ }^{\mathbf{1 8}}$.

A descentralização da autoridade para a implementação de políticas e execução de serviços públicos demanda disponibilidade de recursos para confrontar as responsabilidades alocadas aos demais entes da federação, com participação ativa do governo central no financiamento dessas políticas e serviços ${ }^{19}$. Nesse contexto, as transferências diretas para os governos subnacionais evitam os desequilíbrios entre a disponibilidade de recursos e a oferta de serviços, e são cruciais à implementação das políticas descentralizadas, de tal forma que o impacto para a equidade depende de como as transferências intergovernamentais são realizadas ${ }^{20}$. Assim, ainda que a implementação das políticas e a execução dos serviços sejam descentralizados, o governo central tem papel importante, sendo as transferências intergovernamentais um instrumento valioso para a redução das desigualdades.

Elas também são consideradas relevante mecanismo de coordenação federativa. Abrucio $^{\mathbf{1 3}}$ assinala que a coordenação federativa pode se dar por meio de três mecanismos: i) regras legais de compartilhamento de competências no campo das políticas públicas; ii) estabelecimento de fóruns que contem com a participação dos próprios entes ou nos quais haja representação dos seus interesses; e iii) indução das políticas por parte do governo federal. No âmbito da indução, as transferências intergovernamentais condicionadas cumprem papel relevante na implementação de políticas sociais no Brasil14,21. 
No tocante às possibilidades de o governo central implementar políticas de seu interesse, parece haver predominância na literatura da conclusão de que quanto maior for o número de vetos, maiores seriam as suas dificuldades para isso. Federações subdivididas em vários governos autônomos e com representação desproporcional das unidades que as compõem no Senado, em termos de suas populações, teriam mais frequentemente imposição da vontade da minoria à maioria e apresentariam maiores dificuldades para o governo central implementar políticas públicas nacionais ${ }^{22}$.

Entretanto, segundo Arretche ${ }^{22}$, a federação brasileira desafia tal tese, pois, nesse caso, não se observam constrangimentos à maioria pela minoria, e a autoridade do governo central é favorecida pela competência legislativa abrangente da União, pelo comportamento partidário dos senadores e pela não exigência de maiorias expressivas para a aprovação de matérias de seu interesse. Soares e Machado ${ }^{16}$ concordam com as conclusões de Arretche de que, no Brasil, há concentração de poder na União e acrescentam que a implementação de políticas sociais universais e equitativas é favorecida em modelos federativos como o brasileiro, nos quais há centralização de recursos administrativos e fiscais na União.

No que concerne à implementação da política de saúde em contexto federativo e dado o momento atual de pandemia de Covid-19, a literatura do campo da economia aporta contribuição relevante. De acordo com Costa-Font e Greer ${ }^{23}$, em sistemas de saúde descentralizados, a produção de bens públicos, como os serviços voltados ao combate a epidemias, precisa ser pensada considerando as economias de escala e as externalidades, o que requer oferta com algum grau de centralização desses serviços de saúde. Transpondo esse achado para o contexto atual, pode-se concluir que a natureza dos serviços de vigilância em saúde, as externalidades decorrentes, tanto da pandemia quanto dos serviços públicos oferecidos, e as desigualdades de acesso a serviços de saúde de Média e Alta Complexidade
(MAC) justificam participação mais ativa do governo federal nos esforços de combate ao novo coronavírus.

No Brasil, a esse respeito e de forma mais abrangente, sugeriu-se ao governo federal maior articulação político-institucional e comunicação com a sociedade ${ }^{24}$. Já no campo da saúde, além das ações relativas ao financiamento do SUS e gestão do sistema, destacou-se a necessidade de uma estrutura de governança e coordenação da resposta à pandemia ${ }^{25}$. Considerando que a coordenação dos esforços de enfrentamento da Covid-19 depende fortemente de como se estabelecem as relações intergovernamentais e da efetividade dos mecanismos de coordenação federativa implementados no SUS, a próxima seção apresenta um resumo de evidências da literatura a esse respeito.

\section{Coordenação federativa no SUS}

O SUS foi criado considerando a estrutura federativa definida pela Constituição Federal de 1988 (CF/88), a qual estabeleceu os papéis da União, estados e municípios, atribuindo autonomia administrativa e tributária aos entes de federação. A CF/88 estabelece, também, competências concorrentes, com autorização para qualquer ente implementar políticas sociais em várias áreas ${ }^{26}$. No caso da saúde, a diretriz constitucional estabelece a criação do SUS como sistema descentralizado, com direção única em cada esfera de governo, o que, na prática, resultou em sua implementação por meio de uma relação direta entre a União e os municípios. Esse processo, associado à forma como a federação se organiza, gerou um afastamento dos estados da coordenação federativa do SUS. O que permitiu que isso fosse se conformando ao longo do tempo?

Para responder a essa questão, cinco pontos merecem destaque: 1) a forma como as receitas tributárias são arrecadas e distribuídas; 2) o papel central atribuído à União; 3) os modelos de descentralização de recursos adotados; 
4) as tentativas de retomada do processo de regionalização, mas que ainda apresentam grandes limitações; e 5) as tentativas de instituir instrumentos de coordenação federativa por meio de colegiados de pactuação.

No que se refere à arrecadação direta, os tributos coletados pela União respondem por dois terços do total. Ao mesmo tempo, a CF/88 estabeleceu um conjunto de transferências obrigatórias desses recursos arrecadados pela União, por meio dos chamados fundos de participação, e, também, os tributos arrecadados pelos estados e municípios. Em 2019, os municípios arrecadaram $7,6 \%$ dos tributos e ficaram com $20,7 \%$ do total das receitas tributárias. Os estados perderam parcela de sua arrecadação (27,5\%) nessa redistribuição, ficando com $25 \%$ do total, e a metade restante ficou com a União ${ }^{27}$.

Ao mesmo tempo, a União usou o espaço que lhe foi dado pelo art. $23 \mathrm{da} \mathrm{CF} / 88$, que trata, entre outras questões, da competência comum em matéria de saúde entre os entes federados, para realizar a coordenação federativa por meio da edição de leis e outras normas ${ }^{28}$. Segundo Arretche ${ }^{\mathbf{2 6}}$, esse arranjo federativo deu grande poder de definição da política para a União, a qual optou por exercê-lo por meio de várias portarias do MS.

A estratégia de descentralização da execução de ações e serviços de saúde para municípios ficou clara, principalmente, nas Normas Operacionais Básicas (NOB), particularmente, a NOB $1 / 93$, que introduz a transferência 'fundo a fundo' e a NOB 1/96, que inaugura uma importante fase da política de transferências federais: o incentivo à adoção de programas específicos de natureza nacional. Ainda que a NOB 1/96 tenha proposto resgatar o papel dos estados na coordenação federativa, o que se observa efetivamente é uma ampliação das transferências federais para municípios ${ }^{29}$. Essa descentralização municipalista permitiu avançar no acesso da população a serviços de saúde, principalmente de atenção básica, com importantes impactos sobre os indicadores de saúde da população brasileira ${ }^{\mathbf{3 0} 31}$. Contudo, ainda que a atenção básica possa ser organizada no nível municipal, os serviços mais complexos demandam escala mínima para serem ofertados.

Em 2000, o País já possuía mais de 5.500 municípios, os quais eram, em sua maioria, de pequeno porte (menos de 20 mil habitantes). Além disso, a estrutura de oferta de serviços de MAC era concentrada em alguns municípios de médio e grande porte, o que demanda a coordenação entre eles, assim como com seus estados para garantia de atenção integral à saúde para toda a população. Quanto à organização da rede regionalizada de ações e serviços de saúde, ainda nos anos 2000 , foram várias as tentativas de avançar nesse processo, mas elas ocorreram de forma muito diferenciada. Os ganhos relativos foram maiores onde houve maior investimento ou onde os estados mostraram maior capacidade de coordenar o processo de regionalização $0^{32-34}$.

Nos anos 1990, foram criados colegiados para discussão e pactuação entre os entes subnacionais (Comissão Intergestores Bipartite - CIB) e desses com o gestor nacional (Comissão Intergestores Tripartite - CIT). Nesses colegiados, os atores com poder de veto são ouvidos, e algumas de suas demandas são incorporadas ${ }^{26}$. Entretanto, analisando as atas e as decisões da CIT entre 2000 e 2011, Palotti ${ }^{35}$ observou que $85 \%$ das proposições apresentadas nesse colegiado foram feitas pela União e que as representações de estados e municípios usaram pouco seu poder de veto. Os colegiados pensados para pactuação no âmbito regional têm atuado de forma irregular. Muitos deles têm limitado poder decisório e falta de estrutura e recursos para sua organização. Enfrentam, também, a ausência de recursos para redução da desigualdade de oferta regional de serviços de média e, principalmente, de alta complexidade 32,36 .

A descentralização dos recursos federais em direção aos municípios, com redução dos repasses para os estados (gráfico 1), acabou contribuindo para a fragilização do papel desses últimos na coordenação do sistema em seu território. 
Gráfico 1. Participação das transferências aos estados, das transferências aos municípios e das aplicações diretas no gasto total em ASPS* do Ministério da Saúde (2002 a 2019)

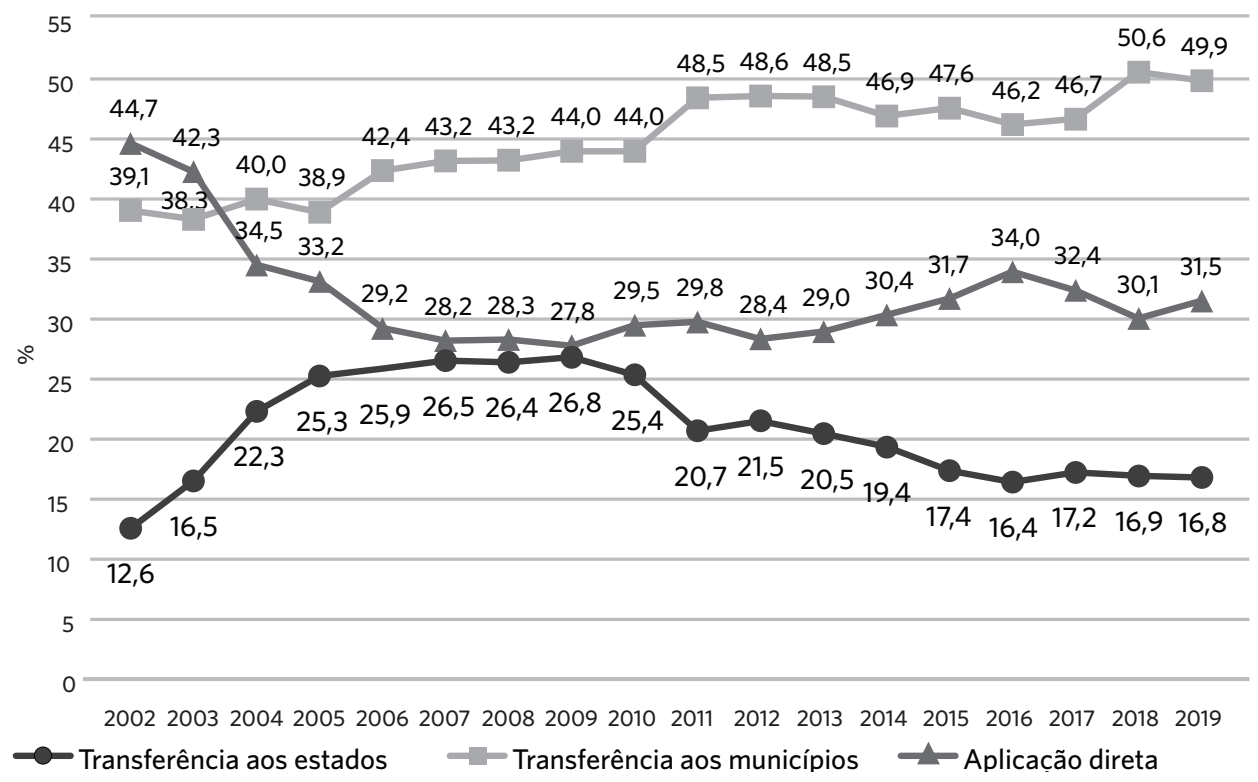

Fonte: Siga Brasi|37. Elaboração própria.

*ASPS: Ações e Serviços Públicos de Saúde.

Em síntese, há evidências de que o governo federal cumpriu papel estratégico na implementação de políticas e programas de saúde no Brasil, valendo-se dos três mecanismos de coordenação federativa apontados pela literatura ${ }^{13,14}$, com forte indução pelo MS, mas com papel secundário delegado aos estados ${ }^{38}$. Entretanto, mudanças envolvendo a atuação federal se operaram recentemente, com consequências para o arranjo de coordenação federativa do SUS.

\section{Fragilização recente dos mecanismos de coordenação federativa do SUS}

Em meados da década passada, a recessão econômica e o forte ressurgimento da agenda neoliberal, em meio à crise política, resultaram em um processo de afastamento do governo federal da coordenação do SUS, o qual pode ser observado pela fragilização dos mecanismos de coordenação federativa instituídos.

O primeiro mecanismo enfraquecido foi o de indução federal, que tem nas transferências intergovernamentais seu maior sustentáculo. Contribuiu para tanto a aprovação da Emenda Constitucional no 95 (EC 95) em 2016, que congela o gasto mínimo obrigatório da União em Ações e Serviços Públicos de Saúde (ASPS) entre 2018 até 2036 no patamar da aplicação mínima de 2017, com evidentes prejuízos para a redução das desigualdades na oferta de serviços no SUS e para a efetivação do direito à saúde no Brasili39.

Mesmo antes da EC 95, em que havia vinculação da aplicação mínima do MS ao crescimento do produto interno bruto (2000 a 2015) e à receita corrente líquida da União (2016 a 
2017), as desigualdades da despesa per capita em ASPS entre as grandes regiões revelam que a alocação de recursos pelo Ministério não foi capaz de equalizar os investimentos em saúde. Houve queda da participação da União no montante de recursos alocados ao SUS entre 2003 e 2017 (de 50,1\% para 43,2\%), e o subfinanciamento do sistema, que é um dos grandes problemas para a sua consolidação, foi agravado com a aprovação da EC 95, pois a tendência é de redução ainda maior da participação federal no seu financiamento ${ }^{40}$.

A EC 95 explicitou, do ponto de vista político, a ausência de compromisso com a garantia de acesso universal, igualitário e integral aos serviços de saúde por meio do SUS, o que pôde ser observado também pelo comportamento do ministro da saúde da época, quando propôs, em 2017, alterações de regulamentação do setor para permitir a oferta de planos de saúde acessíveis, planos mais baratos, com cobertura assistencial limitada a procedimentos ambulatoriais ${ }^{41}$.

No mesmo ano, o MS, com forte defesa pelo Conselho Nacional de Secretarias Municipais de Saúde (Conasems), aprovou na CIT uma proposta que foi chamada de SUS Legal. O objetivo era construir as bases para a desvinculação total dos repasses federais a políticas, programas e serviços. Almejava-se a transferência global dos recursos, o que, no contexto da EC 95, sem a possibilidade de ampliação do orçamento federal para o SUS e com os recursos do orçamento já comprometidos, implicava a limitação e até mesmo eliminação do mecanismo de indução federal de políticas via transferência intergovernamental. Na prática, o propósito inicial não foi alcançado por dificuldades operacionais e legais envolvendo a execução orçamentária federal. No entanto, foi aprovada a flexibilização da execução financeira dos recursos transferidos pelo Ministério para os estados e municípios em dois blocos de financiamento, custeio e investimento ${ }^{42}$.

Outro indicativo da redução do papel do Ministério na coordenação de políticas nacionais no âmbito do SUS é o aumento da execução do orçamento carimbado por emendas parlamentares, para além do que estabelece o orçamento impositivo. Os recursos alocados por deputados e senadores aumentaram significativamente nos últimos anos e têm sido amplamente utilizados no incremento do Piso de Atenção Básica (PAB) e do Teto MAC. Entre 2014 e 2018, passaram de R \$240,1 milhões para $\mathrm{R} \$$ 6,6 bilhões, em valores constantes, apresentando aumento de $2.650 \%$ no período ${ }^{43}$.

No que se refere aos gastos com ações e serviços de vigilância epidemiológica, o que se observou entre 2010 e 2019 foi a ampliação das transferências para os municípios e a redução das transferências para os estados e da aplicação direta do $\mathrm{MS}^{\mathbf{4 4}}$. Isso sugere que houve maior descentralização das responsabilidades envolvendo as ações e serviços de vigilância epidemiológica em direção aos municípios. Porém, há medidas que envolvem a produção de bens públicos e causam externalidades, as quais demandam uma ação centralizada, como apontado anteriormente ${ }^{23}$.

Há necessidade de investimentos nos laboratórios centrais de saúde pública para ampliar a capacidade de testagem e reduzir a subnotificação, aumentando o potencial da vigilância e possibilitando o imediato isolamento dos casos confirmados ${ }^{3}$; de medidas para a ampliação e coordenação da Rede de Atenção à Saúde, com maior participação da Atenção Primária à Saúde (APS) na resposta à pandemia ${ }^{25,45}$, para a compra e o fornecimento de Equipamentos de Proteção Individual (EPI) ${ }^{\mathbf{4 6}}$. A literatura aponta resultados positivos da descentralização por adequar os serviços às necessidades da população e fomentar as capacidades locais, mas ela apresenta também os desafios para garantia da integralidade da atenção à saúde ${ }^{\mathbf{4 7 , 4 8}}$, sendo necessário algum grau de centralização dos serviços ${ }^{48}$.

Com a crise sanitária, inicialmente, o MS realizou ações, principalmente na área de vigilância em saúde, incluindo a declaração de Emergência em Saúde Pública de Importância Nacional, com a criação de grupo executivo interministerial, a publicação de editais para aquisição de EPI e de boletins 
epidemiológicos ${ }^{49}$. Entretanto, sucessivas divergências entre o MS e a Presidência da República geraram impasses e atrasos na aplicação dos recursos federais em ações voltadas ao enfretamento da pandemia ${ }^{50}$. Após a queda do primeiro ministro do governo atual, tornaram-se mais frágeis os outros dois instrumentos de coordenação federativa do SUS: a capacidade de os estados e municípios serem ouvidos nas instâncias de representação e a elaboração de normas para o sistema com a participação da representação das três esferas de governo. Mesmo os recursos extraordinários que suplementaram o orçamento do MS e que poderiam ser transferidos para as secretarias estaduais e municipais de saúde têm tido baixa execução pelo órgão ${ }^{50}$.

Quanto aos outros mecanismos de coordenação, alguns episódios denotam a escalada do conflito na relação tripartite, o não reconhecimento por parte do governo federal da importância das instâncias de representação dos estados e municípios para a gestão do SUS e para a coordenação das ações de resposta à pandemia. Entre eles, o impedimento para que representantes do Conselho Nacional de Secretários de Saúde (Conass) e do Conasems participassem da sessão de posse do segundo ministro da saúde do atual governo ${ }^{51}$; a desconsideração desses conselhos na tomada de decisão de ações para enfrentamento da pandemia, como, por exemplo, quando da publicação de orientação para uso de cloroquina e hidroxicloroquina no tratamento da Covid-1952, sem seguir a norma que estabelece a análise pela Comissão Nacional de Incorporação de Tecnologias em Saúde ${ }^{53}$; a acusação, de indicado do governo para assumir cargo no Ministério, de que os secretários estaduais de saúde tinham interesse em informar mais mortes por Covid-19 do que as que realmente haviam acontecido, a fim de receberem mais recursos ${ }^{54}$; e o lançamento de um painel pelo Conass para apresentação dos números de casos e óbitos por Covid-19, após o MS atrasar a divulgação dos dados e alterar a metodologia de registro dos óbitos ${ }^{55}$.
Sobre a questão federativa neste momento no Brasil, Abrucio et al. ${ }^{56}$ afirmam que o federalismo sob a ótica do governo federal é paradoxal. Os autores enfatizam que o governo federal visa reduzir a participação dos entes subnacionais na deliberação e no controle de decisões tomadas no âmbito da União. Ao mesmo tempo que prega a necessidade de maior autonomia para os entes, toma decisões que os afetam sem sua participação. Ainda segundo esses autores, esse processo está na base da descoordenação da política de saúde que se presencia em meio à pandemia de Covid-19, o que, obviamente, acarreta graves consequências, como se discute a seguir.

\section{A coordenação federativa e os desafios para resposta à pandemia}

Chegamos a 2020 em um processo de ampliação da descentralização no SUS, ainda sem os instrumentos de coordenação regional consolidados e com uma mudança na visão do governo federal sobre o seu papel como coordenador nacional da política. Quando o coronavírus entrou no País, já havia muitas limitações para enfrentá-lo, e a atuação federal se impõe como um grande desafio.

Ainda convivemos com grandes desigualdades no acesso a serviços de MAC. Em uma pandemia que velozmente demandou leitos de Unidades de Terapia Intensiva (UTI) e respiradores, logo ficou evidente essa desigualdade no acesso aos serviços ${ }^{57-59}$. Considerando a oferta existente e os cenários de taxas de contágio, estados do Norte e do Nordeste enfrentariam colapsos em seus sistemas de saúde rapidamente. Contudo, outra realidade logo se impôs: as diferenças de acesso entre grupos da população mais vulneráveis e aqueles com mais alta renda. Mesmo em estados com maior oferta de serviços de saúde, o acesso a eles é desigual. Essa é uma questão importante no enfrentamento da doença, dado que o tempo 
para acesso a um serviço de saúde com capacidade suficiente para garantir a atenção é fator central para reduzir os riscos de morte nos casos mais graves de Covid-1960.

Para que o sistema de saúde tivesse capacidade de se organizar, vários países do mundo adotaram medidas de distanciamento social para minimizar os espaços de contato e contágio pelo coronavírus. Mesmo em países como a Alemanha, que havia ampliado seus investimentos em leitos de UTI e respiradores, essas medidas foram implementadas ${ }^{61}$. No Brasil, contudo, não houve decisão do governo federal para adotá-las em âmbito nacional. Elas ficaram a cargo dos estados e dos municípios, que as adotaram de maneira pouco coordenada e com efetividades variadas ${ }^{62}$.

Ainda nos primeiros momentos, houve problemas para realização das compras públicas de equipamentos de proteção individual, essenciais para prevenir o contágio entre profissionais de saúde. Com a escassez internacional desses equipamentos, compras ou negociações centralizadas com fornecedores poderiam ter tido mais êxito e custo mais baixo ${ }^{\mathbf{4 6}}$.

Também houve perda de protagonismo do Ministério, com implicações no cancelamento de compras de respiradores e contratação de leitos de UTI ${ }^{63}$, além de convocações pelo Congresso para o ministro interino prestar esclarecimentos sobre as ações do órgão ${ }^{64}$. Os estados utilizaram de vários mecanismos para adquirir os equipamentos; e, em alguns casos, houve 'guerra sanitária', com disputa entre os entes e, também, desses com o setor privado ${ }^{65,66}$, mas também espaço para a cooperação horizontal entre os estados ${ }^{67}$.

Adicionalmente, a resposta da vigilância em saúde para controle dos casos e prevenção do contágio, que também depende da coordenação federal, não foi satisfatória. Essa coordenação é necessária para: elaboração de protocolos nacionais para notificação dos casos; organização da vigilância em locais de potencial contágio (portos, aeroportos, terminais rodoviários); busca ativa de casos a partir de outros notificados; e elaboração e divulgação de boletins epidemiológicos para reduzir as falhas de comunicação com a sociedade e com a imprensa, gerar alertas locais, bem como informar a população sobre os locais de maior incidência da doença. Ademais, para a organização dos laboratórios centrais de saúde pública e das unidades notificadoras, para o contato permanente com os outros serviços de saúde e orientação das compras públicas, entre outras atividades.

Outro ponto relevante é a importância da atenção primária na rede de cuidados e vigilância dos casos para identificar localidades e populações de risco para a Covid-19 e, também, manter o acompanhamento de outros problemas de saúde da população em seus territórios ${ }^{\mathbf{2 5}, 45}$. Recente pesquisa nacional com profissionais e gestores municipais de saúde aponta que a atenção primária tem buscado se reinventar durante a pandemia com o uso de instrumentos de atendimento à distância e visitas peridomiciliares dos Agentes Comunitários de Saúde (ACS). Porém, aponta também oferta insuficiente de EPI e de capacitação para os profissionais, além de elevada proporção de ACS realizando recepção a sintomáticos nas unidades de saúde e menor proporção realizando visita peridomiciliar ou a distância. Os autores do estudo destacam a necessidade de investir em ações que permitam solucionar esses problemas, ampliando a participação da APS na resposta à pandemia ${ }^{68}$.

$O$ fato de os estados entrarem fortemente no processo de coordenação da resposta à pandemia fortaleceu seu papel no SUS e ampliou o espaço do Conass. Todavia, esse protagonismo foi assumido com grande dificuldade, pois, como discutido neste ensaio, no decorrer da implantação do SUS, eles perderam espaço na coordenação regional do sistema e há uma assimetria de poder na federação em favor da União. Além disso, os estados enfatizaram a gestão dos serviços de alta complexidade, especialmente daqueles oferecidos nas unidades de saúde próprias, e pouco se articularam com os municípios para a oferta de leitos em âmbito regional ou na articulação da rede de serviços com o envolvimento da APS. 
Se por um lado a ascensão da participação dos estados se mostra positiva e relevante, por outro, ficou clara a importância de uma coordenação tripartite. A coordenação deficiente dos esforços de enfrentamento da Covid-19 resulta em ineficiências no SUS e no comprometimento da efetividade das ações de vigilância em saúde e de assistência à saúde. Além disso, cobra um alto preço da população brasileira que pode ser, no extremo, um elevado número de óbitos evitáveis, com custo intangível das vidas perdidas.

\section{Considerações finais}

Em síntese, até meados da década passada, a trajetória da coordenação federativa no SUS revelava uma construção assentada em forte atuação federal, com o uso dos três mecanismos de coordenação, em especial, a indução da implantação de programas e serviços por meio da transferência de recursos financeiros para os estados e municípios. A partir de então, o recrudescimento da agenda neoliberal no governo federal resultou em um processo de distanciamento do MS do seu papel de dirigente nacional do sistema, com consequente fragilização dos mecanismos de coordenação do SUS.

A crise sanitária provocada pela pandemia de Covid-19 acelerou esse processo e explicitou a decisão do governo atual pela inação, relegando ao MS um papel secundário nos esforços de enfrentamento da crise. A coordenação federal deficiente na resposta à pandemia não se deve à ausência de mecanismos de coordenação federativa do SUS ou ao constrangimento desses mecanismos imposto pelos demais entes da federação. Como visto anteriormente, no federalismo brasileiro, a União detém muito poder; e isso não mudou. O que se observa não é a perda de sua capacidade para coordenar os esforços do SUS, mas, sim, a deliberada fragilização dos mecanismos de coordenação federativa pelo governo federal, que se acentuou nos últimos meses.

Com isso, pode-se dizer que, se houver um projeto de saúde para o País, ele não é o SUS, pois, para a consolidação do SUS constitucional, a utilização de mecanismos para a coordenação federativa do sistema é imprescindível. Além disso, no contexto da pandemia, a opção federal pela inação resulta em graves consequências para o funcionamento do sistema, para $o$ atendimento das necessidades de saúde da população brasileira e para a efetividade das ações de enfrentamento da doença no País.

\section{Aviso legal}

As opiniões emitidas neste artigo são de exclusiva e inteira responsabilidade das autoras, não exprimindo, necessariamente, o ponto de vista da instituição à qual se vinculam.

\section{Colaboradoras}

Vieira FS (0000-0001-7377-7302)* e Servo LMS (0000-0003-0770-7378)* contribuíram igualmente para a elaboração do manuscrito. 


\section{Referências}

1. World Health Organization. Influenza and COVID-19 similarities and differences [internet]. Geneva: WHO; 2020. [acesso em 2020 jun 10]. Disponível em: https://bit.ly/3hbr781.

2. Bastos LS, Niquini RP, Lana RM, et al. COVID-19 e hospitalizações por SRAG no Brasil: uma comparação até a $12^{\mathrm{a}}$ semana epidemiológica de 2020 . Cad. Saúde Pública. 2020; 36(4):e00070120.

3. França EB, Ishitani LH, Teixeira RA, et al. Óbitos por COVID-19 no Brasil: quantos e quais estamos identificando? Rev. bras. epidemiol. 2020; (23):e200053.

4. Vieira FS. Implicações de decisões e discussões recentes para o financiamento do Sistema Único de Saúde. Saúde debate. 2016; 40(109):187-199.

5. Santos NR. SUS 30 anos: o início, a caminhada e o rumo. Ciênc. Saúde Colet. 2018; 23(6):1729-1736.

6. Fleury S. Pandemia, contradições e inovações no federalismo brasileiro [internet]. Rio de Janeiro: CEE Fiocruz; 2020. [acesso em 2020 jun 24]. Disponível em: https://bit.ly/2VqUODZ.

7. Duczmal LH, Almeida ACL, Duczmal DB, et al. Vertical social distancing policy is ineffective to contain the COVID-19 pandemic. Cad. Saúde Pública. 2020; 36(5):e00084420.

8. UOL. Bolsonaro fala em onda enorme de desemprego e "põe no colo" de governadores. UOL. 2020 jun 7. [acesso em 2020 jun 10]. Disponível em: https:// bit.ly/2UsAaba.

9. Zylberkan M, Campos JP. Queda de dois ministros em apenas um mês deixa Saúde à deriva. Veja. 2020 maio 2. [acesso em 2020 jun 10]. Disponível em: https://bit.ly/3f7nb6z.

10. Brasil. Ministério da Saúde. Orientações do Ministério da Saúde para manuseio medicamentoso precoce de pacientes com diagnóstico da covid-19 [internet]. Brasília, DF: Ministério da Saúde; 2020. [acesso em
2020 jun 10]. Disponível em: https://bit.ly/2XOSRYr.

11. Chowdhury MS, Rathod J, Gernsheimer JA. Rapid Systematic Review of Clinical Trials Utilizing Chloroquine and Hydroxychloroquine as a Treatment for COVID-19. Acad Emerg Med [internet]. 2020 [acesso em 2020 jul 10]; 27(6):493-504 Disponível em: http:// doi: 10.1111/acem.14005.

12. Brasil. Supremo Tribunal Federal. STF reconhece competência concorrente de estados, DF, municípios e União no combate à Covid-19 [internet]. Brasília, DF: STF; 2020. [acesso em 2020 jun 10]. Disponível em: https://bit.ly/37kiovR.

13. Abrucio FL. A coordenação federativa no Brasil: a experiência do período FHC e os desafios do governo Lula. Rev. Sociol. Polit. 2005; (24):41-67.

14. Lima LD. A coordenação federativa do sistema público de saúde no Brasil. In: Fundação Oswaldo Cruz. A saúde no Brasil em 2030: organização e gestão do sistema de saúde. v.3 Rio de Janeiro: Fiocruz; Brasília, DF: Ipea; Ministério da Saúde; 2013. p. 73-139.

15. Guest JL, Del Rio C, Sanchez T. The three steps needed to end the covid-19 pandemic: bold public health leadership, rapid innovations, and courageous political will. JMIR Pub. Health Surv. 2020; 6(2):e19043.

16. Soares MM, Machado JA. Federalismo e políticas públicas [internet]. Brasília, DF: Enap; 2018. [acesso em 2020 jun 11]. Disponível em: https://bit.ly/3cS2Lgc.

17. Souza C. Políticas públicas: questões temáticas e de pesquisa. Caderno CRH. 2003; (39):11-24.

18. Costa-Font J, Greer SL. Health system federalism and decentralization: what is it, why does it happen, and what does it do? In: Costa-Font J, Greer SL. Federalism and decentralization in European health and social care. London: Palgrave Macmillan; 2013. p. 1-9.

19. Leite VR, Vasconcelos CM, Lima KC. Federalism and decentralization: impact on international and Brazi- 
lian health policies. Int J Health Serv. 2011; 41(4):711723.

20. Abimbola S, Baatiema L, Bigdeli M. The impacts of decentralization on health system equity, efficiency and resilience: a realist synthesis of the evidence. Health Polic. Plan 2019; 34(8):605-617.

21. Viana ALA, Machado CV. Descentralização e coordenação federativa: a experiência brasileira na saúde. Ciênc. Saúde Colet. 2009; 14(3):807-817.

22. Arretche M. Quando instituições federativas fortalecem o governo central? In: Hochman G, Faria CAP, organizadores. Federalismo e políticas públicas no Brasil. Rio de Janeiro: Fiocruz; 2013. p. 65-90.

23. Costa-Font J, Greer SL. Territory and health: perspectives from economics and political science. In: Costa-Font J, Greer SL. Federalism and decentralization in European health and social care. London: Palgrave Macmillan; 2013. p. 13-43.

24. Schimidt F, Mello J, Cavalcante P. Estratégias de coordenação governamental na crise da covid-19 [internet]. Brasília, DF: Ipea; 2020. (Nota Técnica Diest n. 32). [acesso em 2020 jun 10]. Disponível em: https:// bit.ly/3e2Klfe.

25. Massuda A, Malik AM, Ferreira Junior WC, et al. Pontos-chave para gestão do SUS na resposta à pandemia covid-19 [internet]. São Paulo: IEPS; 2020. (Nota Técnica n. 6). [acesso em 2020 jun 10]. Disponível em: https://bit.ly/37l34PK.

26. Arretche M. Federalismo e políticas sociais no Brasil: problemas de coordenação e autonomia. São Paulo Perspec. 2004; 18(2):17-26.

27. Afonso JR, Castro KP. Carga tributária bruta 2019: recorde histórico e evidências federativas [internet]. JRRA. 2020 mar 13[acesso em 2020 jun 25]. Disponível em: https://bit.ly/2Vmyi3X.

28. Machado JA, Palotti PLM. Entre cooperação e centralização: federalismo e políticas sociais no Brasil pós-1988. Rev. bras. Ci. Soc. 2015; 30(88):61-82.
29. Piola SF, Vianna SM, Marinho A, et al. Saúde no Brasil: algumas questões sobre o Sistema Único de Saúde (SUS) [internet]. Brasília DF: Ipea; 2009. (Texto para Discussão n. 1391). [acesso em 2020 jun 25]. Disponível em: https://bit.ly/2ZgsqdG.

30. Paim J, Travassos C, Almeida C, et al. O sistema de saúde brasileiro: história, avanços e desafios. The Lancet. 2011; (1):11-31.

31. Castro MC, Massuda A, Almeida G, et al. Brazil's unified health system: the first 30 years and prospects for the future. The Lancet. 2019; 394(10195):345-356.

32. Lima LD, Viana ALA, Machado CV, et al. Regionalização e acesso à saúde nos estados brasileiros: condicionantes históricos e político-institucionais. Ciênc. Saúde Colet. 2012; 17(11):2881-2892.

33. Menicucci T, Marques AMF. Cooperação e coordenação na implementação de políticas públicas: o caso da saúde. Dados. 2016; 59(3):823-865.

34. Servo LMS. O processo de regionalização da saúde no Brasil: acesso geográfico e eficiência [tese]. Belo Horizonte: Universidade Federal de Minas Gerais; 2020.

35. Palotti PLM, Machado JA. Coordenação federativa e a "armadilhada decisão conjunta": as comissões de articulação intergovernamental das políticas sociais no Brasil. Dados. 2014; 57(2):399-441.

36. Moreira MR, Ribeiro JM, Ouverney AM. Obstáculos políticos à regionalização do SUS: percepções dos secretários municipais de Saúde com assento nas Comissões Intergestores Bipartites. Ciênc. Saúde Colet. 2017; 22(4):1097-1108.

37. Brasil. Senado Federal. Siga Brasil. Sistema de informações sobre orçamento público federal [internet]. [acesso em 2020 jun 11]. Disponível em: https://bit. ly/30t3y4a.

38. Andrade MV, Noronha K, Sá EB, et al. Desafios do sistema de saúde brasileiro. In: Di Negri JA, Araújo BCPO, Bacelette RG. Desafios da nação: artigos de apoio [internet]. v. 2. Brasília, DF: Ipea; 2018. 
[acesso em 2020 jun 11]. Disponível em: https://bit. ly/2Vc38eH.

39. Vieira FS, Benevides RPS. Os impactos do Novo Regime Fiscal para o financiamento do Sistema Único de Saúde e para a efetivação do direito à saúde no Brasil [internet]. Brasília, DF: Ipea; 2016. (Nota Técnica Disoc n. 28). [acesso em 2020 jun 11]. Disponível em: https://bit.ly/3hm36Lu.

40. Piola SF, Benevides RPS, Vieira FS. Consolidação do gasto com ações e serviços públicos de saúde: trajetória e percalços no período de 2003 a 2017 [internet]. Rio de Janeiro: Ipea; 2018. (Texto para Discussão n. 2439). [acesso em 2020 jun 10]. Disponível em: https://bit.ly/30qQlcN.

41. Sá EB. O público e o privado no sistema de saúde: uma apreciação do projeto de Plano de Saúde Acessível. Brasília, DF: Ipea; 2018. [acesso em 2020 jun 11]. Disponível em: https://bit.ly/3fgpTql.

42. Jaccoud L, Vieira FS. Federalismo, integralidade e autonomia no SUS: desvinculação da aplicação de recursos federais e os desafios da coordenação. Rio de Janeiro: Ipea; 2018. (Texto para Discussão n. 2399). [acesso em 2020 jun 10]. Disponível em: https://bit. ly/2zqLjlu.

43. Piola SF, Vieira FS. As emendas parlamentares e a alocação de recursos federais no Sistema Único de Saúde. Rio de Janeiro: Ipea; 2019. (Texto para Discussão n. 2497). [acesso em 2020 jun 12]. Disponível em: https://bit.ly/2Ynlivy.

44. Vieira FS, Benevides RPS. Gasto federal com vigilância em saúde na última década e necessidade de reforço do orçamento do Ministério da Saúde para enfrentamento à pandemia pelo coronavírus. Brasília, DF: Ipea; 2020. (Nota Técnica Disoc n. 61). [acesso em 2020 jun 22]. Disponível em: https://bit.ly/37Olgin.

45. Tasca R, Massuda A. Estratégias para reorganização da Rede de Atenção à Saúde em resposta à pandemia COVID-19: a experiência do sistema de saúde italiano na região de Lazio. APS em Rev. 2020; 2(1):20-27.
46. Servo LMS, Ramos MCB, Peters JR, et al. Equipamentos de proteção individual, higienizantes e material de higiene pessoal: preços, regulação e gestão da informação em tempos de coronavírus. Brasília, DF: Ipea; 2020. (Nota Técnica Disoc n. 63). [acesso em 2020 jun 25]. Disponível em: https://bit.ly/2Vly0dw.

47. Lima LD, Queiroz LFN, Machado CV, et al. Descentralização e regionalização: dinâmica e condicionantes da implantação do Pacto pela Saúde no Brasil. Ciênc. Saúde Colet. 2012; 17(7):1903-1914.

48. Ribeiro JM, Moreira MR, Ouverney AM, et al. Políticas de saúde e lacunas federativas no Brasil: uma análise da capacidade regional de provisão de serviços. Ciênc. Saúde Colet. 2017; 22(4):1031-1044.

49. Croda JHR, Garcia LP. Resposta imediata da Vigilância em Saúde à epidemia da COVID-19. Epidemiol. Serv. Saúde. 2020; 29(1):e2020002.

50. Afonso JR, Pinto EG. A velha falta de prioridade e uma nova tragédia anunciada. Poder 360. 2020 abr 28. [acesso em 2020 jun 12]. Disponível em: https:// bit.ly/2Atrp9R.

51. Brasilino CE. Conselhos de secretários de Saúde são barrados na posse de Teich: Ministro enviou ofício aos presidentes do Conass e do Conasems onde pede desculpas pelo constrangimento ocorrido na cerimônia no Planalto[internet]. Metrópoles. 2020 abr 17. [acesso em 2020 jun 12]. Disponível em: https://bit. ly/3cUwYLQ.

52. Conselho Nacional de Secretários de Saúde. Nota oficial: "Orientações do Ministério da Saúde para tratamento medicamentoso precoce de pacientes com diagnóstico da Covid-19" [internet]. Brasília, DF: Conass; 2020. [acesso em 2020 jun 12]. Disponível em: https://bit.ly/3fnPBtf.

53. Brasil. Lei $\mathrm{n}^{\circ}$ 12.401, de 28 de abril de 2011. Altera a Lei $n^{\circ} 8.080$, de 19 de setembro de 1990, para dispor sobre a assistência terapêutica e a incorporação de tecnologia em saúde no âmbito do Sistema Único de Saúde - SUS [internet]. 2011. [acesso em 2020 jun 12]. Disponível em: https://bit.ly/2MOuwf6. 
54. Conselho Nacional de Secretários de Saúde. CONASS repudia acusação de manipulação de dados sobre Covid-19 [internet]. Brasília, DF: Conass; 2020. [acesso em 2020 jun 12]. Disponível em: https://bit. ly/2zu58Z6.

55. Conselho Nacional de Secretários de Saúde [internet]. Painel Conass: covid-19. Brasília, DF: Conass; 2020. [acesso em 2020 jun 12]. Disponível em: https://bit. ly/37rYQpw.

56. Abrucio LA, Grin EJ, Couto C, et al. Mais Brasil, Menos Brasília: o sentido do federalismo bolsonarista e seu impacto no combate à covid-19. Estadão. 2020 abr 12. [acesso em 2020 jun 10]. Disponível em: https://bit.ly/37nfl6e.

57. Noronha KVMS, Ramalho GG, Maldonado TC, et al. The COVID-19 pandemic in Brazil: analysis of supply and demand of hospital and ICU beds and mechanical ventilators under different scenarios. Cad. Saúde Pública. 2020; 36(6):e00115320.

58. Rache B, Rocha R, Nunes L, et al. Para além do custeio: necessidades de investimento em leitos de UTI no SUS sob diferentes cenários da Covid-19 [internet]. São Paulo: Instituto de Estudos para Políticas de Saúde; 2020. (Nota Técnica n. 7). [acesso em 2020 jun 25]. Disponível em: https://bit.ly/2Ntr9um.

59. Castro MC, Carvalho LR, Chin T, et al. Demand for hospitalization services for COVID-19 patients in Brazil. medRxiv. 20201 abr. No Prelo.

60. Pereira RHM, Braga CKV, Servo LMS, et al. Mobilidade urbana e o acesso ao Sistema Único de Saúde para casos suspeitos e graves de covid-19 nas vinte maiores cidades do Brasil [internet]. Brasília, DF: Ipea; 2020. (Nota Técnica Dirur n. 14). [acesso em 2020 jun 25]. Disponível em: https://bit.ly/2Z50MQI.

61. Sá EB. A saúde pública e o enfrentamento da crise causada pelo coronavírus. Brasília, DF: Ipea; 2020. (Nota Técnica Disoc n. 68). [acesso em 2020 jun 25]. Disponível em: https://bit.ly/2Nw4Kgl.
62. Moraes R. Medidas legais de incentivo ao distanciamento social: comparação das políticas e governos estaduais e prefeituras das capitais no Brasil [internet]. Brasília, DF: Ipea; 2020. (Nota Técnica Dinte n. 16). [acesso em 2020 jun 25]. Disponível em: https:// bit.ly/2BGqcMM.

63. Bertoni E. Os atrasos e omissões de um Ministro da Saúde provisório [internet]. Nexo Jornal. 2020 jun 1. [acesso em 2020 jun 25]. Disponível em: https://bit. ly/3duYRdp.

64. Brasil. Senado Federal. Comissão da covid-19 ouve ministro da Saúde na terça [internet]. Agência Senado. 2020 jun 22. [acesso em 2020 jun 25]. Disponível em: https://bit.ly/2BCG8zC.

65. Associação Nacional de Hospitais Privados. COVID-19: Entidades do setor de Saúde pedem medidas urgentes ao STF contra abusos de autoridade [internet]. São Paulo: ANAPH; 2020. [acesso em 2020 jun 25]. Disponível em: https://bit.ly/2AlraTa.

66. Godoy M, Venceslau P, Galhardo R. Confisco de luvas a respiradores opõe União e estados. Estado de Minas. 2020 mar 27. [acesso em 2020 jun 25]. Disponível em: https://bit.ly/2Vf6PB6.

67. Grin EJ. Cooperação federativa entre os estados no Brasil: efeitos imprevistos do covid-19? [internet]. Estadão 2020 abr 4. [acesso em 2020 jun 26]. Disponível em: https://bit.ly/31vVAZl.

68. Bousquat A, Giovanella L, Medina MG, et al. Desafios da Atenção Básica no enfrentamento da pandemia da Covid-19 no SUS. Relatório de Pesquisa [internet]. Rio de Janeiro: Rede de Pesquisa em APS Abrasco; 2020. [acesso em 2020 set 2]. Disponível em: https://bit.ly/2ForweO.

Recebido em 27/06/2020

Aprovado em 28/09/2020

Conflito de interesses: inexistente

Suporte financeiro: não houve 